\title{
Diarrhea in the HIV-infected patient
}

PETER PHILLIPS MD FRCPC

\begin{abstract}
P PHILliPS. Diarrhea in the HIV-infected patient. Can J Infect Dis 1994;5(Suppl E):42E-48E. Gastrointestinal manifestations may be encountered throughout the course of HIV disease. The problems range from asymptomatic hairy leukoplakia to overwhelming diarrhea due to opportunistic infections such as cryptosporidiosis. Diarrheal disease is an important contributing factor to wasting in advanced HIV disease. Considerable progress has been made in recents years regarding our understanding of HIV-related diarrhea, including etiological agents, diagnostic methods and treatment.
\end{abstract}

Key Words: AIDS, Diarthea, Enteric pathogens, Human immunodeficiency virus

\section{Diarrhée chez le patient infecté par le VIH}

RÉSUMÉ : Les manifestations gastro-intestinales peuvent s'observer tout au cours de la maladie au viH. Les problèmes varient d'une leucoplasie à cellules chevelues asymptomatique à une diarrhée considérable due aux infections opportunistes, comme la cryptosporidiose. La maladie diarrhéique est un important facteur contributif dans les états de déperdition observés dans la maladie au viH avancée. Des progrès considérables ont été accomplis au cours des dernières années dans notre compréhension de la diarrhée liée au VIH, y compris au chapitre des agents étiologiques. des méthodes diagnostiques et des thérapeutiques.

$\mathrm{D}$ SEASES RESULTING IN GASTROINTESTINAL TRACT SYMPtoms are common and occur in up to $50 \%$ of patients with AIDS (1). Diarrhea is the most frequent symptom in such patients; others include oral and esophageal symptoms, nausea, vomiting, abdominal pain, jaundice and wasting. A wide range of intestinal infections may account for diarrheal illness in the HIVinfected patient. These pathogens include bacteria, (Salmonella, Shigella, Campylobacter and Yersinia species and Clostridium difficile), viruses (cytomegalovirus [CMv], adenovirus), mycobacteria (Mycobacterium avium, Mycobacterium tuberculosis), and protozoa (Cryptosporidium species, Giardia lamblia, Entamoeba histolytica, Isospora belli and microsporidia).

Three types of enteric infections include the following (2):

- Noninflammatory processes involving primarily the proximal portion of the small bowel as a result of an enterotoxin (eg, enterotoxigenic Escherichia coli) or infection that interferes with small bowel absorption (eg, cryptosporidium, giardia, rotavirus, Norwalk-like viruses, CMV, $M$ avium and possibly HIV).

- Inflammatory dysentery, which involves the colon as a result of invasive infection, possibly including a cytotoxin (eg, Shigella, Salmonella and Campylobacter species, $C$ difficile or amebiasis). Included in this category of disease are the various causes of sexually transmitted proctitis (eg, herpes simplex virus, gonococcus, chlamydia and Treponema pallidum).

- An enteric fever syndrome, which may occur with bacteremic illness, often associated with constipation early in the course of the disease (eg, Salmonella species, and occasionally campylobacter or yersinia).

Patients presenting with the noninflammatory diarrhea involving predominantly small bowel tend to have soft to watery bowel movements, which may be associated with midabdominal discomfort, in the absence of

Division of Infectious Diseases, St Pauls Hospital; and the University of British Columbia, Vancouver, British Columbia

Correspondence: Dr Peter Phillips, Pacific Region, Canadian HIV Clinical Trials Network, 210-1033 Davie Street, Vancouver, British Columbia V6B 1 M7 
blood or mucus per rectum. In contrast, inflammatory dysentery involving the colon or rectum usually results in frequent, smaller volume diarrhea, with or without lower abdominal pain, tenesmus, rectal urgency and blood or mucus per rectum. Fecal leukocytes are usually absent in noninflammatory diarrhea, but are often present in inflammatory diarrhea involving the colon or rectum; however, the sensitivity of fecal leukocyte smears for the diagnosis of inflammatory diarrhea is unclear, and has not been evaluated in HIV-infected patients.

The commonly identified causes of chronic diarrhea in AIDS patients include cryptosporidiosis, microsporidiosis, isosporiasis, or intestinal involvement with $M$ avium or cytomegalovirus. No specific etiology is found in 30 to $50 \%$ of cases of HIV-related chronic diarrhea. By exclusion, these cases have been diagnosed as idiopathic HIV enteropathy (3), although the diagnostic criteria for this entity have been debated (4), and the etiological significance of other enteric viruses such as astrovirus and picobirnavirus has not been determined in HIV-infected patients (5).

\section{CRYPTOSPORIDIOSIS}

Cryptosporidiosis is a zoonosis. Both animal to person and person to person transmission have been documented. Risk factors for cryptosporidiosis include deficient cellular or humoral immunity, infancy, close contact with infected individuals, travel to developing countries, poor sanitary facilities and occupational exposure (animal workers and day care centre employees) (6). The infection has also been documented to be waterborne. The two recognized species are Cryptosporidium parvum and Cryptosporidium muris.

Infection of the gastrointestinal tract with cryptosporidium is one of the most common causes of chronic diarrhea in AIDS patients (7) and accounts for considerable morbidity.

A significantly higher mortality rate has been associated with AIDS patients who have cryptosporidiosis compared with those who do not, suggesting that the infection often results in general deterioration (8). The prevalence of cryptosporidiosis is 3 to $4 \%$ for AIDS patients in the United States compared with prevalence as high as 50\% in Haiti and Africa (7). The disease may affect both the normal and immunocompromised host and usually results in self-limited and chronic disease, respectively.

Clinical presentation includes diarrhea that is often severe and ranges up to 25 bowel movements per day. The stools are watery, voluminous (up to $20 \mathrm{~L} /$ day) and not associated with blood or inflammatory cellular exudate. Frequently there is marked weight loss, and both lactose intolerance and fat malabsorption have been documented in association with cryptosporidiosis. In the normal host the infection is usually self-limited with symptoms resolving within two weeks, but stools remain positive for the organism for an additional two to three weeks (9). Chronic cryptosporidiosis lasting longer than one month in patients without other causes of immunodeficiency is an AIDS-defining illness. Among HIV-infected individuals with cryptosporidiosis, selflimited disease has been associated with higher CD4 cell counts (mean 312 cells $/ \mathrm{mm}^{3}$ ) compared with those cases of persistent infection (mean CD4 counts 57 cells/ $\mathrm{mm}^{3}$ ) (10). Flanigan et al (10) observed spontaneous resolution of cryptosporidiosis in all patients $(n=8)$ with CD4 counts greater than 180 cells $/ \mathrm{mm}^{3}$; however, 34 of $39(87 \%)$ cases with CD4 counts less than 180 cells/ $\mathrm{mm}^{3}$ had persistent disease. Symptoms of cryptosporidiosis tend to wax and wane even without treatment (11).

Cryptosporidiosis has been shown pathologically to involve all portions of the gastrointestinal tract from the pharynx to the rectum including the biliary tree. Most often the disease is largely confined to the small and large intestine. Extraintestinal sites of infection have included the biliary tract, pancreatic duct, liver and lung (6). The pathology of cryptosporidiosis is similar to that seen in giardiasis, including small intestinal changes with epithelial loss, villous atrophy, crypt elongation and often minimal inflammatory infiltrate in the adjacent lamina propria $(6,12)$.

Diagnosis is most readily made by stool smears which may be stained using a number of techniques including modified acid-fast stain, fluorescent auraminerhodamine stain, periodic acid-Schiff and carbolfuchsin stains (7). The modified acid-fast stain is generally the preferred method for diagnosis. Methods for concentrating stool samples are usually not required for diagnosis in acute cases. A sodium chloride layering technique followed by ethyl acetate sedimentation has been shown to be superior to other concentration methods (13). Radiographs of the bowel in patients with cryptosporidiosis usually show nonspecific findings including prominent mucosal folds, thickened intestinal wall and disordered motility. Intestinal biopsy is generally considered to be less sensitive than stool examinations because of the patchy nature of the disease and the absence of gross signs of inflammation to help direct the endoscopist. Intestinal biopsy may reveal the presence of 4 to $5 \mu \mathrm{m}$ diameter organisms, which are adherent to the epithelial surface on the microvilli and which are contained in a vacuole that is considered intracellular, but extracytoplasmic to the enterocyte (7).

Management of cryptosporidiosis in AIDS has been mainly supportive $(6,7,9)$. There are now some potentially beneficial antiparasitic agents, but none proven effective in randomized, placebo controlled, comparative trials. Oral and sometimes intravenous fluid replacement may be required. Antimotility agents are not always helpful and in some patients may be associated with increased abdominal cramping (7). Intravenous hyperalimentation is helpful for stabilizing occasional patients but may be inappropriate for terminally ill patients, and is further limited by cost considerations 
TABLE 1

Treatment of diarrhea in the HIV-infected patient

Type of infection

Cryptosporidiosis

Preferred treatment

symptomatic treatment with nutritional supplements, antidiarrheal agent (eg

operamide, diphenoxylate), \pm fluid (iv/po) and electrolyte replacement

\section{Isosporiasis}

Acute infection TMP-SMX 1 DS tablet gid $\times 10$ days ${ }^{5}$

Symptomatic treatment with nutritional

supplements, antidiarrheal agent (eg

operamide, diphenoxylate), \pm fluid (po/iv)

and electrolyte replacement

Suppressive

treatment

TMP-SMX $]$ DS tablet $3 x /$ week

Microsporidiosis

Symptomatic treatment with nutritional supplements, antidiarrheal agents (eg, operamide, diphenoxylate) \pm fluid (po/iv) and electrolyte replacement

Cytomegalovirus Ganciclovir $5 \mathrm{mg} / \mathrm{kg}$ iv bid $x$ 14-21 days colitis

Salmonella

Bacteremia

Ampicillin $2 \mathrm{~g}$ iv every $4-6 \mathrm{~h} \times 1-4$ weeks

then amoxicillin $500 \mathrm{mg}$ tid to complete

2-4 week course

or

Ciprofloxacin $750 \mathrm{mg}$ po every $12 \mathrm{~h} \times 2-4$ weeks

Enterocolitis Ampicillin $500 \mathrm{mg}$ po gid x 1-2 weeks (if susceptible)

or

TMP-SMX 1 DS tablet bid $\times$ 1-2 weeks

Shigella Ciprofloxacin $500 \mathrm{mg} \mathrm{po} \mathrm{bid} \times 7$ days

colitis

Clostridium difficile Metronidazole $250 \mathrm{mg}$ po qid $\mathrm{x} 10$ days colitis
Alternative treatment

Experimental agents:

Paromomycin (EDRP) $500 \mathrm{mg}$ po gid with food

$x$ 14-28 days, then $500 \mathrm{mg}$ po bid

- Azithromycin (EDRP) $1200 \mathrm{mg}$ po first day, then

$600 \mathrm{mg} /$ day $\times 27$ days, then $300 \mathrm{mg} /$ day

Octreotide (Sandostatin) 50-500 $\mu \mathrm{g} \mathrm{sc}$ tid for

symptomatic therapy of intractable diarrhea

Pyrimethamine $50-75 \mathrm{mg}$ po/day + folinic acid

5-10 mg/day $\times 1$ month

Pyrimethamine $25 \mathrm{mg}+$ sulfadoxine $500 \mathrm{mg}$

(1 Fansidar tablet (EDRP)) once weekly

Pyrimethamine $25 \mathrm{mg}$ + folinic acid $5 \mathrm{mg} / \mathrm{day}$

Experimental agents:

Albendazole $400 \mathrm{mg}$ po bid (EDRP)

- Metronidazole 500 mg po tid

Foscarnet $60 \mathrm{mg} / \mathrm{kg}$ iv every $8 \mathrm{~h} \times 14-21$ days

or $100 \mathrm{mg} / \mathrm{kg}$ iv every $12 \mathrm{~h} \times 14-21$ days)

Cefotaxime 1-2 g iv every 6-8 h

Ceftriaxone 1-2 g iv daily

\section{Ciprofloxacin 500 mg po bid}

Norfloxacin 400 mg po bid

TMP-SMX 1 DS po bid

Vancomycin $125 \mathrm{mg}$ po gid $\times 10$ days
Comments

No controlled trials to show efficacy of antimicrobials.

Spiramycin ineffective in non-HIV infants ${ }^{3}$ Numerous

anecdotal but unproven treatments. Nutritional

supplements often required for severe cases, + parenteral hyperalimentation

Spontaneous resolution unlikely if absolute CD4 count $<200 / \mathrm{mm}^{3}$

Duration of high dose TMP-SMX therapy is not well defined

Duration is not well defined

Efficacy of albendazole and metronidazole not established Modified trichrome stain optimal for detecting

microsporidia in stool and duodenal aspirates

Efficacy not clearly established for syndromes other than retinitis. Some evidence for efficacy in colitis Ganciclovir preferred if renal impairment; foscarnet preferred if neutropenia or AZT used concurrently

Need for maintenance therapy remains controversial except for CMV retinitis

Recurrent bacteremia may be suppressed by ciprofloxacin $500 \mathrm{mg}$ po bid or TMP-SMX 1 DS bid indefinitely

Unlike normal host, $\mathrm{HIV}^{+}$patient requires treatment of uncomplicated salmonella enterocolitis to reduce complication rate (eg, bacteremia)

Long term ciprofloxacin, norfloxacin or TMP-SMX if recurrent shigellosis

C difficile colitis more common in $\mathrm{HIV}^{+}$patients. Similar efficacy ${ }^{12}$ but vancomycin more expensive than metronidazole. For severe infection: vancomycin 250-500 $\mathrm{mg}$ po every $6 \mathrm{~h}$. Either drug unreliable iv for $C$ difficile colitis. Try to decrease responsible antibiotic(s). Avoid antimotility agents. Relapse in up to $20 \%$ 
and potential complications. It is believed that the biliary tree may serve as a reservoir for cryptosporidiosis and thereby contributes to recurrent infections because of difficulty in eradicating the organism from this site. A wide range of specific treatments has been employed for cryptosporidiosis $(6,7,9,14)$ with some reported clinical and microbiological responses. Favourable responses have been observed in association with paromomycin (15), bovine hyperimmune colostrum (16), and letrazuril (17).

An important aspect of management is reducing transmission by good hygiene including hand washing and awareness of the risks of direct fecal-oral exposure (9). Nosocomial spread of this infection has been documented.

\section{ISOSPORIASIS}

Isosporiasis is an uncommon cause of chronic diarrhea in HIV-infected individuals in North America (less than $0.2 \%$ ) but has been observed in up to $15 \%$ of AIDS patients in Haiti. It is clinically indistinguishable from cryptosporidiosis, typically presenting with chronic, watery diarrhea without blood or mucus, and associated with crampy abdominal pain, nausea and weight loss (18). Stool smears are usually negative for fecal leukocytes, and the diagnosis is established by stool smears (modified acid-fast stain or iodine preparation) demonstrating the 25 to $30 \mu \mathrm{m}$ diameter oocysts, which are larger than the $5 \mu \mathrm{m}$ cryptosporidial cysts. Other means of establishing the diagnosis include duodenal aspirate or biopsy in which organisms are demonstrated within the cytoplasm of villous epithelium. In contrast to cryptosporidiosis, most patients respond promptly to treatment (Table 1), but relapse occurs in approximately 50\% without secondary prophylaxis (18).

\section{MICROSPORIDIOSIS}

Microsporidiosis is an intestinal protozoal infection caused by Enterocytozoon bieneusi. It has been found to be an important cause of chronic diarrhea in HIVinfected patients when routine stool microbiology has been negative (12). Microsporidia are spore-forming, obligate, intracellular protozoan parasites, and the organism resides in the cytoplasm of the intestinal epithelial lining cells. The clinical presentation is indistinguishable from that of cryptosporidiosis, with watery, nonbloody diarrhea associated with progressive weight loss and usually no fever. Previously the diagnosis was usually only established by duodenal or jejunal biopsy with the parasite identified on hematoxylin and eosin or Giemsa stain. Electron microscopy has been used to confirm the diagnosis, although in a recent study, light microscopy appeared to be of similar sensitivity (19). Recently, a practical method using a modified trichrome stain was reported for the identification of the spores of $E$ bieneusi in fecal samples (20). Optimal treatment for intestinal microsporidiosis has not been established, but responses have been reported with metronidazole as well as albendazole (Table 1) $(21,22)$. However, patients with microsporidia infection may not have diarrhea. This observation prompted Rabeneck et al (23) to question the role of microsporidia in the pathogenesis of HIV-related chronic diarrhea.

\section{CYTOMEGALOVIRUS COLITIS}

CMV disease may occur at any site of the gastrointestinal tract in HIV-infected patients, but most often affects the colon, esophagus, stomach or hepatobiliary system. CMV colitis occurs in approximately 5 to $10 \%$ of AIDS patients, and is usually associated with chronic diarrhea, abdominal pain, anorexia, weight loss and fever (24). Diarrhea is usually nonbloody, although complications may include gastrointestinal hemorrhage or perforation. Diagnosis is most reliably established by the presence of CMV inclusions on intestinal biopsy specimens, and is supported by the presence of CMV antigen or positive biopsy viral culture for cMV. The absence of other intestinal pathogens is also helpful in establishing the relationship of gastrointestinal complaints to positive findings for CMV. The efficacy of antiviral therapy for CMV disease has not been documented as clearly for colitis as it has for retinitis. A recent placebo controlled trial showed that ganciclovir treated patients had improvement in mucosal abnormalities seen on colonoscopy and some protection against development of new extraintestinal CMV disease, compared with placebo. However, clinical endpoints (eg, diarrhea, body weight, etc) did not differ between the groups (25). Diarrhea response may have been confounded by the use of antidiarrhea agents in both arms of the study. The need for long term maintenance therapy with ganciclovir or foscarnet has not been established, but should at least be offered to those individuals experiencing frequent relapses, which appear to respond to treatment.

\section{MYCOBACTERIUM AVIUM}

$M$ avium infection occurs in 30 to $50 \%$ of AIDS patients, usually beginning with a localized gastrointestinal infection (duodenum, small or large bowel) and frequently followed by dissemination. Macrophages are infected and pathological involvement mainly involves the reticuloendothelial system, including lymph nodes, liver, spleen, bone marrow and gastrointestinal tract (26). The clinical presentation of disseminated $M$ avium infection often includes fevers, night sweats, weight loss and progressive anemia. The presence of abdominal pain and diarrhea without evidence for other enteric pathogens suggests specific gastrointestinal tact involvement, which is often associated with mesenteric lymphadenopathy. Intestinal involvement may result in a malabsorption syndrome, similar clinically and pathologically to Whipple's disease except for the presence of macrophages laden with acid-fast bacilli 


\section{Management of diarrhea in the immunocompromised $(1)^{*}$ HIV-infected patient}

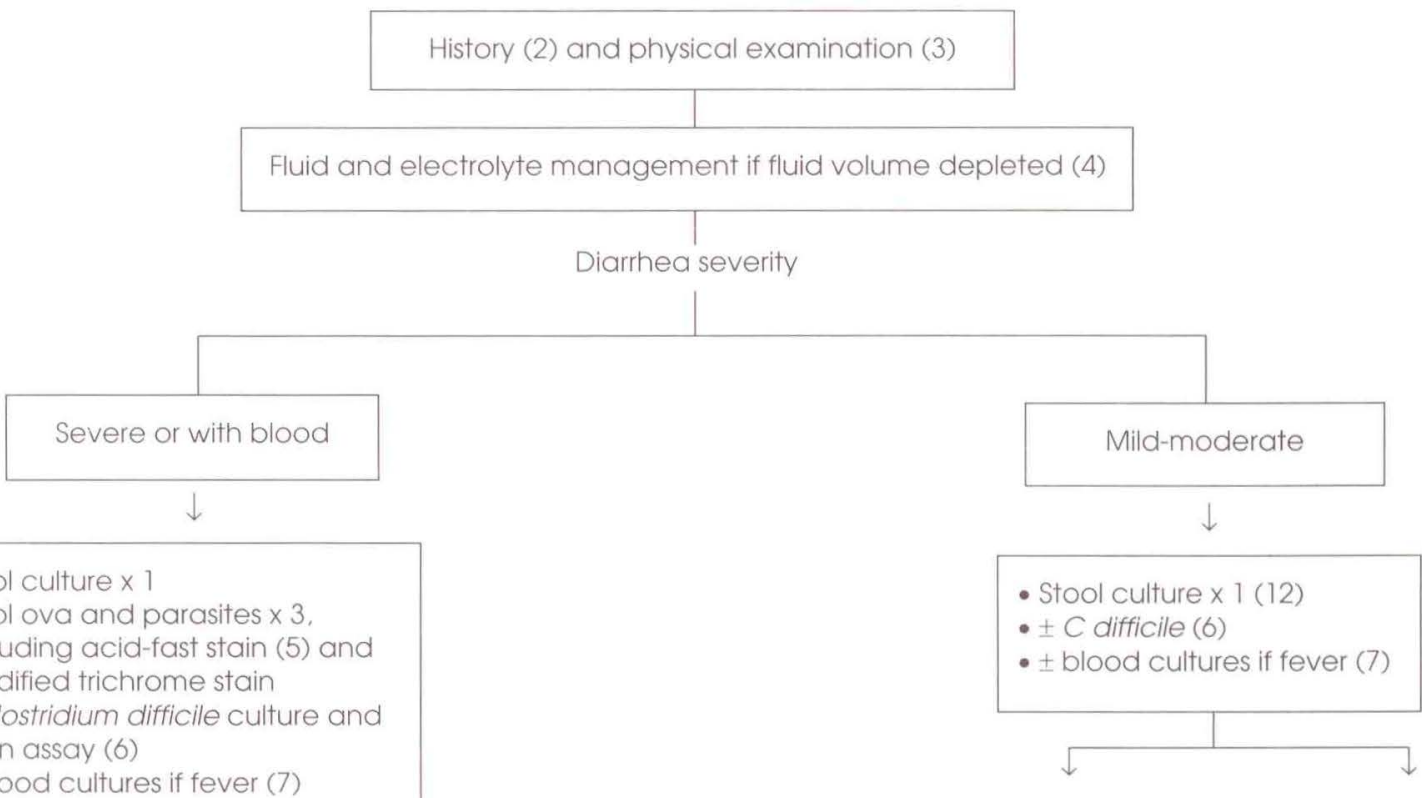

- Stool culture x 1

- Stool ova and parasites $\times 3$

including acid-fast stain (5) and

modified trichrome stain

- \pm Clostridium difficile culture and

toxin assay (6)

- \pm blood cultures if fever (7)

$\downarrow$

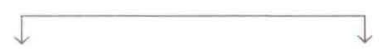

Diagnosis

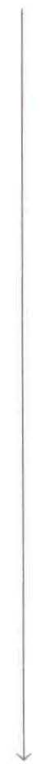

$\downarrow$

$\downarrow$

Response

No diagnosis

Antidiarrheal agent (8)

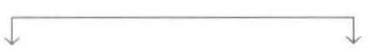

Blood in stool or persistent diarrhea

$$
\downarrow
$$

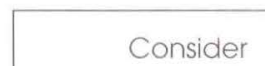
sigmoidoscopy (9) \pm upper gastrointestinal endoscopy (10)

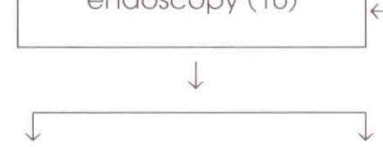

No diagnosis Diagnosis

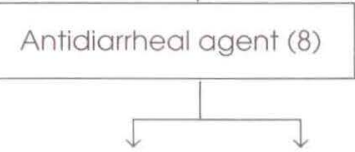

Persistent diarrhea

Response

$\downarrow$

Stool ova and parasites

(5) $\times 3$, including acidfast and modified trichrome stain

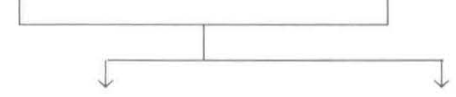

No diagnosis

Diagnosis

Specific treatment

Specific treatment

Diagnosis

No diagnosis
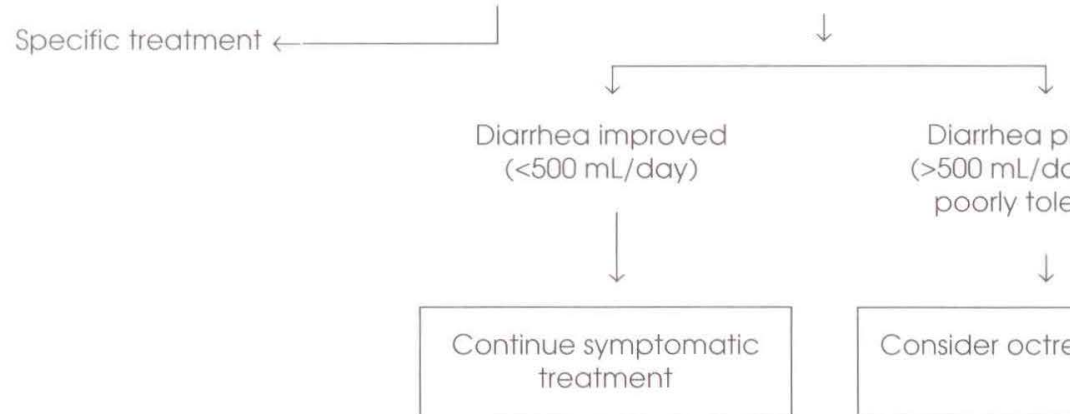

Diarrhea profuse

(>500 mL/day) and

poorly tolerated

$\downarrow$

Consider octreotide (11)

Figure 1) *Please see Footnotes to Figure 1 on following page 
(27). Endoscopic biopsy and mycobacterial culture are most reliable for establishing the diagnosis. The role of stool smears for acid-fast bacilli in the diagnosis of either intestinal or disseminated infection is unclear because of variable sensitivity, and the possibility of mycobacterial colonization and symptoms related to other etiologies.

\section{BACTERIAL ENTERIC PATHOGENS}

Bacterial enteric pathogens should be considered in patients with inflammatory diarrhea; however, approximately half of salmonellosis cases present with fever in the absence of colitis. Salmonellosis (28) and possibly shigellosis (29) are more likely to be associated with bacteremia in the HIV-infected patient than the general population. Unlike the immunocompetent host, all HIV patients with uncomplicated salmonella gastroenteritis require antimicrobial therapy. Recurrent bacterial enteric infections require long term suppressive therapy (Table 1). C difficile colitis, which appears to be more common in HIV patients, should be considered in those

\section{FOOTNOTES TO FIGURE 1}

1. Opportunistic infections involving the gastrointestinal tract, such as Mycobacterium avium or cytomegalovirus (CMv) usually occur in patients with absolute CD4 counts less than $100 / \mathrm{mm}^{3}$.

2. Inquire regarding:

- use of diarrhea-inducing drugs and caffeinated beverages:

- recent antibiotic use (Clostridium difficile)

- Sexual orientation (homosexual men at risk for proctitis due to herpes simplex virus, gonococcus, chlamydia and syphilis);

- ingestion of inadequately cooked seafood (eg, vibrio, Norwalk-like viruses):

- travel to tropical areas (eg, enterotoxigenic Escherichia coli, giardia, Entamoeba histolytica, strongyloides, Norwalk-like viruses or rotavirus and invasive bacterial infections);

- bloody diarrhea (eg, E coli, amebiasis, campylobacter, cMv, shigella)

3. Physical examination should include assessment of intravascular volume, including supine/ standing blood pressure and pulse, and jugular venous pressure.

4. Initial management for fluid volume-depleted patients should be oral or intravenous fluids and electrolytes. A simple oral rehydration solution consists of: one level teaspoon of table salt, plus four heaping teaspoons of sugar, added to $1 \mathrm{~L}$ of water (J Trop Med Hyg 1981;84:73). Give volume equivalent to 5 to $7 \%$ of body weight for mild-moderate dehydration.

5. Stool acid-fast staining is needed for identification of cryptosporidium and Isospora belli. However, stool smear for acid-fast bacilli not routinely recommended because of variable results of sensitivity and specificity for true mycobacterial infection versus colonization. Positive stool smear may be more likely than mycobacterial stool culture to reflect invasive infection rather than colonization. receiving antimicrobial agents for prophylaxis or treatment of various opportunistic infections.

\section{AIDS ENTEROPATHY}

Idiopathic AIDS-associated enteropathy has been defined as chronic diarrhea (longer than one month) associated with negative investigations for enteric pathogens, with or without the demonstration of villous atrophy. The negative studies should include stool microbiology, endoscopy and biopsies, as outlined in Figure 1. Possible etiologies for AIDS-associated enteropathy include: previously unrecognized or seldom isolated enteric pathogens (5); low grade bacterial overgrowth possibly due to impaired development of gut B lymphocytes (related to CD4 lymphocte depletion) and immunoglobulin A production (1); or HIV (30).

\section{MANAGEMENT}

Management of HIV-related diarrhea is summarized in the algorithm (Figure 1). Treatment of specific, selected enteric pathogens is summarized in Table 1 (31).
Modified trichrome stain is the optimal method for light microscopy identification of microsporidia in stool and duodenal aspirate sample (N Engl J Med 1992;326:161).

Entamoeba histolytica is a nonpathogenic commensal in most infected homosexual men ( N Engl J Med 1986:315: 353 ), and rarely causes invasive colitis in AIDs patients.

6. If recent antibiotic use, then also include $C$ difficile culture and toxin assay.

7. Routine blood cultures should be obtained in patients with fever and diarrhea to exclude bacteremia due to salmonella, shigella and campylobacter. Salmonellosis is 20 times more common in AIDS patients and five times more likely to be associated with bacteremia than in the general population ( J Infect Dis 1987;156:998).

Mycobacterial blood cultures are indicated if persistent or recurrent fever develops in association with CD4 Iymphopenia.

8. Antidiarrheal agent of choice is loperamide (Imodium), which is not associated with narcotic dependency, but this may occur with diphenoxylate (Lomotil) (Gastroenterology 1980;79:1272). Diarrhea and abdominal cramps respond earlier with loperamide than bismuth subsalicylate (JAMA $1986 ; 255: 757)$. Antimotility agents should usually be avoided in patients with fever or bloody stools, because they may worsen dysentery due to shigella (JAMA 1973;226:1525) or C difficile (JAMA 1976;235:1454). Loperamide dosing: $4 \mathrm{mg}$ initially, then $2 \mathrm{mg}$ after each unformed stool (maximum $16 \mathrm{mg} /$ day). When daily dose established, it may be given as one to four divided doses/day.

9. Sigmoidoscopy specimens should include wet mount (E histolytica). Biopsies are obtained for pathology, viral ( $\mathrm{CMv}$, adenovirus, herpes simplex virus) and mycobacterial culture. Barium enema and colonoscopy are seldom useful for the investigation of chronic diarrhea in HIV-infected patients (AIDS 1990;4:687).

10. Upper gastrointestinal endoscopy for duodenal fluid specimens should be sent promptly for parasitology (wet 
mount, acid-fast and modified trichrome stains), and biopsies for hematoxylin and eosin, acid-fast, \pm Giemsa stains looking primarily for protozoa (microsporidia, isospora, giardia), mycobacteria and CMV. Electron microscopy may be helpful for diagnosis of microsporidiosis but is expensive and usually not necessary (Ninth International Conference on AIDS, 1993. Abst WS-B20-2). A specimen may be prepared for electron microscopy but not examined unless other specimens are nondiagnostic.

11. Octreotide (Sandostatin) is a synthetic analogue of somatostatin, and in doses of 50 to $500 \mu \mathrm{g}$ subcutaneously tid may provide benefit in severe refractory AIDS-associated watery diarrhea, particularly when no pathogens have been identified (Ann Intern Med 1991:115:705).

12. Initial investigation of mild to moderate AIDS-related diarrhea should be limited to a stool culture (Ann Intern Med 1990;1 12:942). More extensive investigations, which are both expensive and may be associated with patient discomfort, should be reserved for those with a negative stool culture and persistent diarrhea despite symptomatic antidiarrheal treatment.

Reproduced with permission from reference 31

\section{REFERENCES}

1. Smith PD, Quinn TC, Strober W, Janoff EN, Masur H. Gastrointestinal infections in AIDS. Ann Intern Med 1992:116:63-77.

2. Guerrant RL. Bobak DA. Bacterial and protozoal gastroenteritis. N Engl J Med 1991:325:327-40.

3. Greenson JK, Belitsos PC, Yardley JH, Bartlett JG. AIDS enteropathy: Occult enteric infections and duodenal mucosal alterations in chronic diarrhea. Ann Intern Med 1991:114:366-72.

4. Ullrich R, Riecken E-O, Zeitz M. AIDS enteropathy. Ann Intern Med 1991;1 15:328.

5. Grohmann GS, Glass RI, Pereira HG, et al. Enteric viruses and diarrhea in HIV-infected patients. N Engl J Med 1993:329:14-20.

6. Crawford, FG, Vermund SH. Human cryptosporidiosis. Crit Rev Microbiol 1988;16:113-48.

7. Soave R, Johnson WD. Cryptosporidium and Isospora belli infections. J Infect Dis 1988;157:225-9.

8. Navin TR, Hardy AM. Cryptosporidiosis in patients with AIDS. J Infect Dis 1987;115:150. (Lett)

9. Souve R, Armstrong D. Cryptosporidium and cryptosporidiosis. Rev Infect Dis 1986;8:1012-21

10. Flanigan T, Whalen C. Turner J, et al. Cryptosporidium infection and CD4 counts. Ann Intern Med 1992:116:840-2.

11. Cryptosporidiosis: assessment of chemotherapy of males with acquired immune deficiency syndrome (AIDS). MMWR 1982:31:589.

12. Kotler DP, Francisco A, Clayton F. Scholes JV, Orenstein JM. Small intestinal injury and parasitic diseases in AIDS. Ann Intern Med 1990;1 13:444-9.

13. Weber R. Bryan RJ, Juranek DD. Improved stool concentration procedure for detection of cryptosporidium oocysts in fecal specimens. Interscience Conference on Antimicrobial Agents and Chemotherapy. Chicago, 1991. (Abst 656)

14. Wittenberg DF Miller NM, Van den Ende J, et al. Spiramycin is not effective in treating cryptosporidium diarrhea in infants: results of a double-blind randomized trial. J Infect Dis 1989;159:131-2.

15. Gathe J, Plot D, Bernal A, Clemmons J, Stool E. The effectiveness of paromomycin in the treatment of gastrointestinal cryptosporidiosis. Seventh International Conference on AIDS, Florence, 1991. (Abst MB 2270)

16. Nord J, Ma P, Dijohn D, Tzipori S, Tacket CO. Treatment with bovine hyperimmune colostrum of cryptosporidial diarrhea in AIDS patients. AIDS 1990;4:581-4.

17. Harris M, Deutsch G, MacLean JD, Tye L, Tsoukas CM. A phase I study of letrazuril in AIDS-related cryptosporidiosis. Ninth International Conference on

AIDS. Berlin, 1993. (Abst WS-Bl3-5)

18. DeHovitz JA, Pape JW, Boncy M, Johnson WD. Clinical manifestations and therapy of Isospora belli infection in patients with the acquired immunodeficiency syndrome. N Engl J Med 1986;315:87-90.

19. Peacock CS, Blanshard C, Tovey DG, Ellis DS, Gazzard BG. Histological diagnosis of intestinal microsporidiosis in patients with AIDS. J Clin Pathol 1991;44:558-63.

20. Weber R, Bryan RT, Owen RL, Wilcox CM, Gorelkin L, Visvesvara GS, and the Enteric Opportunistic Infections Working Group. Improved light-microscopical detection of microsporidia spores in stool and duodenal aspirates. N Engl J Med 1992;326:161-6.

21. Eeftinck Schatternkerk JKM, van Gool T, van Ketel RJ, et al. Clinical significance of small-intestinal microsporidiosis in HIV-infected individuals. Lancet 1991:337:895-8.

22. Blanshard C, Ellis DS, Tovey DG, Dowell S, Gazzard BG. Treatment of intestinal microsporidiosis with albendazole in patients with AIDS. AIDS 1992;6:311-3.

23. Rabeneck L, Gyorkey F, Genta RM, Gyorkey P, Foote LW, Risser JMH. The role of microsporidia in the pathogenesis of HIV-related chronic diarrhea. Ann Intern Med 1993; 119:895-9.

24. Drew LW. Cytomegalovirus infection in patients with AIDS. Clin Infect Dis 1992;14:608-15.

25. Dieterich DT, Kotler DP, Busch DF, et al. Ganciclovir treatment of cytomegalovirus colitis in AIDS: a randomized, double-blind, placebo-controlled multicenter study. J Infect Dis 1993;167:278-82.

26. Klatt EC, Jensen DF, Mayer PR. Pathology of Mycobacterium avium-intracellulare infection in acquired immunodeficiency syndrome. Hum Pathol 1987:18:709-14.

27. Gray JR, Rabeneck L. Atypical mycobacterial infection of the gastrointestinal tract in AIDS patients. Am J Gastroenterol 1989:84:1521-4.

28. Celum CL, Chaisson RE, Rutherford GW, Barnhart JL, Echenberg DF. Incidence of salmonellosis in AIDS patients. J Infect Dis 1987;156:998-1001.

29. Baskin DH, Lax JD, Barenberg D. Shigella bacteremia in patients with acquired immune deficiency syndrome. Am J Gastroenterol 1987;82:338-41.

30. Ullrich R, Zeitz M, Heise W, Lage M, Hoffken G, Riecken EO. Small intestinal structure and function in patients infected with human immunodeficiency virus (HIV): Evidence for HIV-induced enteropathy. Ann Intern Med 1989;111:15-21.

31. Comprehensive Care Guide for Persons with HIV disease. Ottawa: Health Canada and the College of Family Physicians of Canada, 1993. 


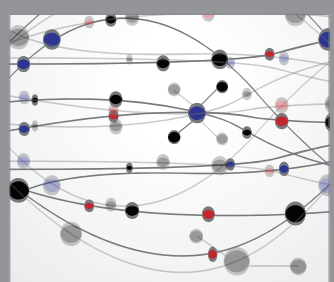

The Scientific World Journal
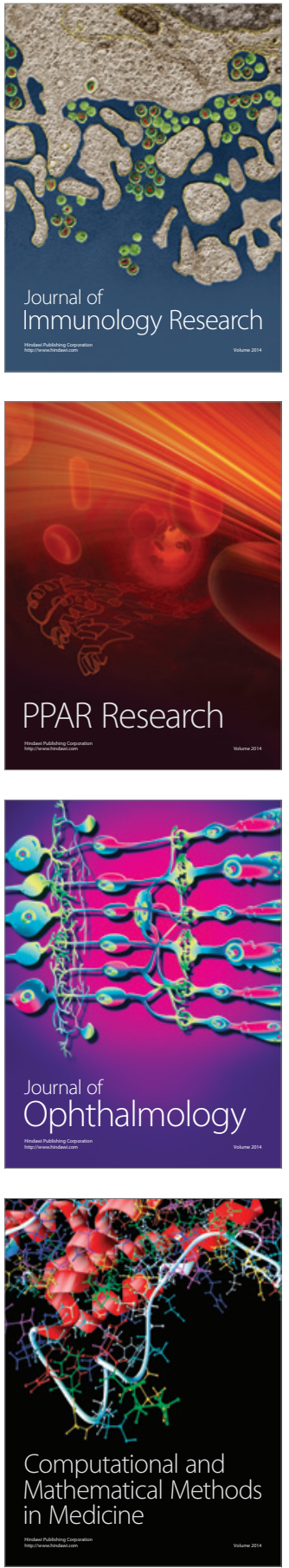

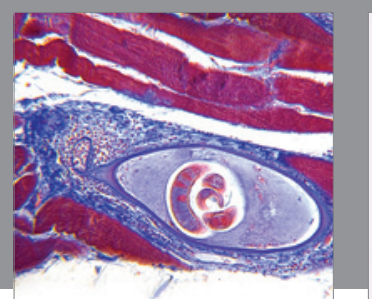

Gastroenterology Research and Practice

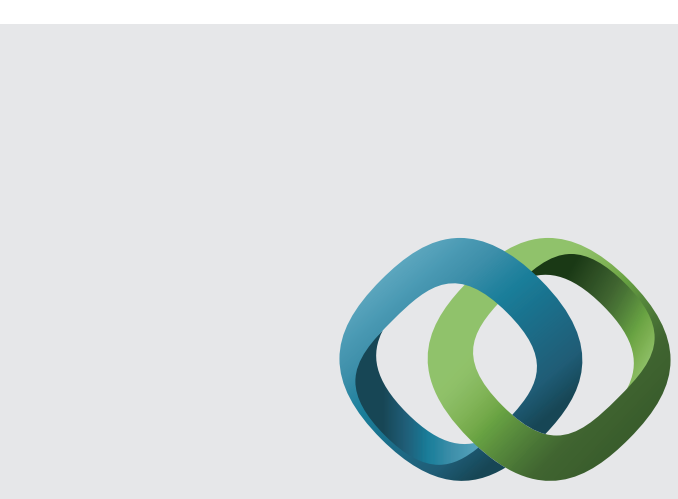

\section{Hindawi}

Submit your manuscripts at

http://www.hindawi.com
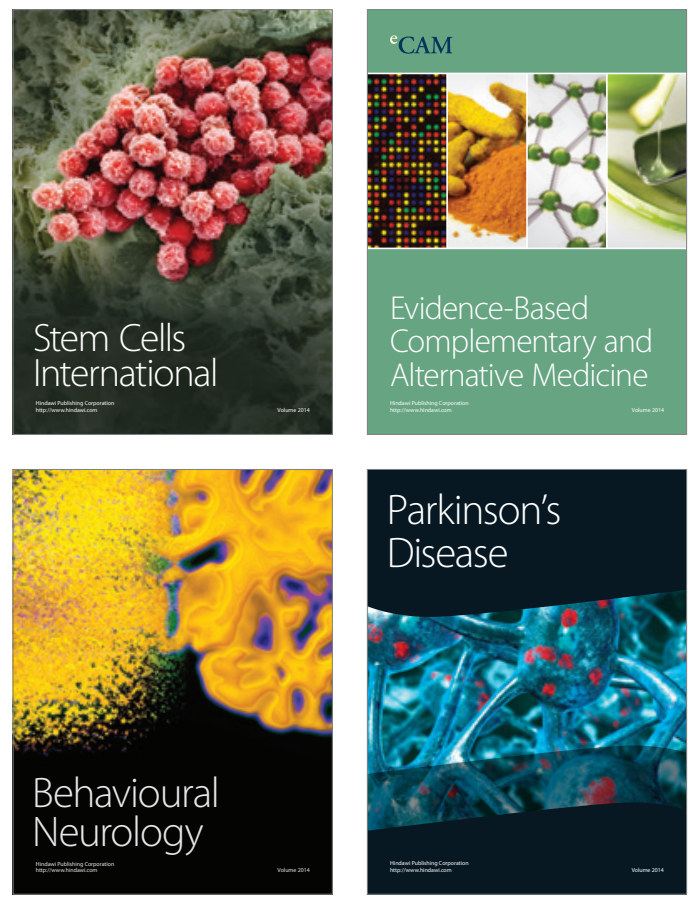
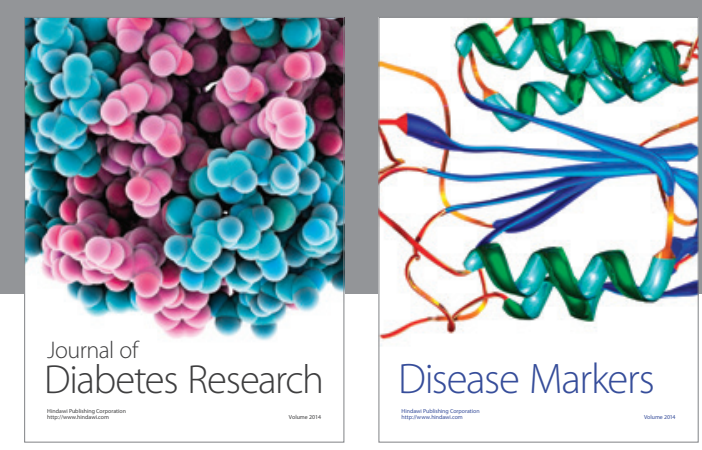

Disease Markers
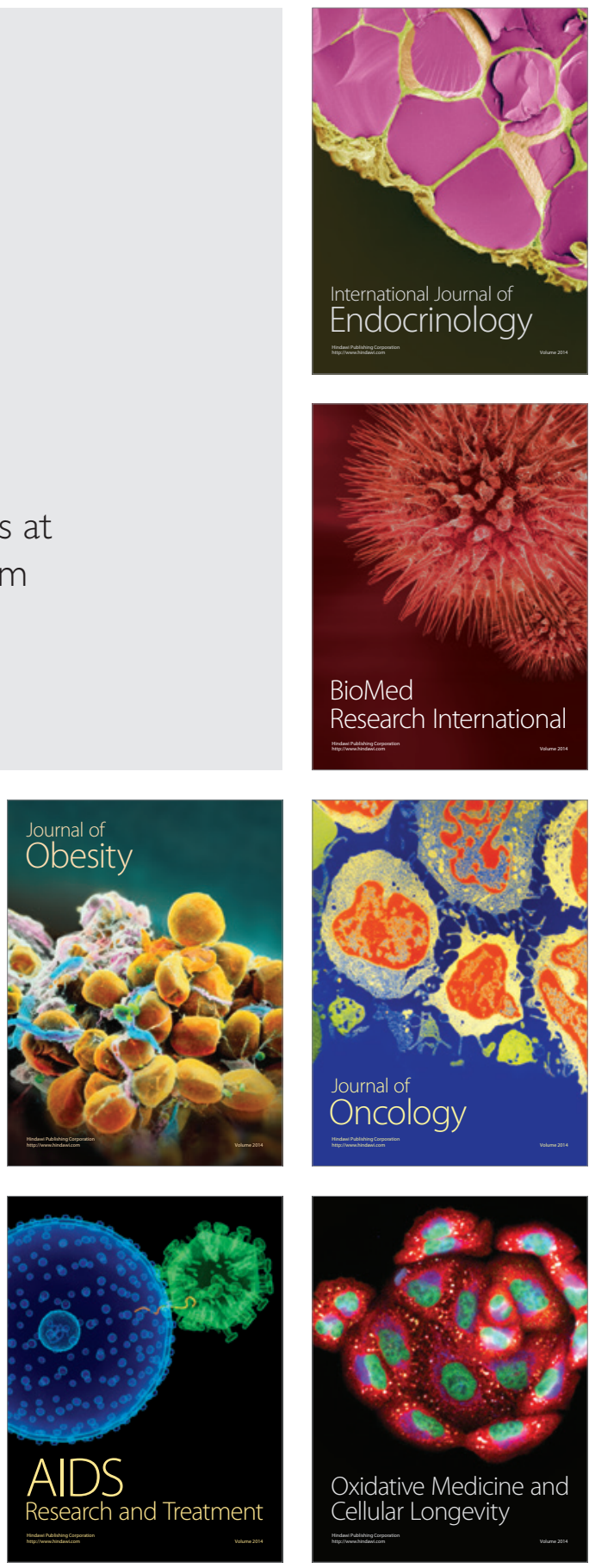